\title{
Context and Aims of the Aqua-Planet Experiment
}

\author{
Michael BLACKBURN \\ National Centre for Atmospheric Science, University of Reading, Reading, UK \\ and
}

Brian J. HOSKINS

Grantham Institute for Climate Change, Imperial College, London, UK

Department of Meteorology, University of Reading, Reading, UK

(Manuscript received 14 September 2011, in final form 17 August 2012)

\begin{abstract}
The Aqua-Planet Experiment (APE) was first proposed by Neale and Hoskins (2000a) as a benchmark for atmospheric general circulation models (AGCMs) on an idealised water-covered Earth. The experiment and its aims are summarised, and its context within a modelling hierarchy used to evaluate complex models and to provide a link between realistic simulation and conceptual models of atmospheric phenomena is discussed. The simplified aqua-planet configuration bridges a gap in the existing hierarchy. It is designed to expose differences between models and to focus attention on particular phenomena and their response to changes in the underlying distribution of sea surface temperature.
\end{abstract}

Keywords comparison of atmospheric general circulation models (GCMs); modelling hierarchy; tropical circulation; intertropical convergence zone (ITCZ); precipitation

\section{Introduction}

The Aqua-Planet Experiment (APE) was proposed by Neale and Hoskins (2000a) as a coordinated comparison of atmospheric general circulation model (AGCM) simulations on a water-covered Earth. Eight idealised distributions of sea surface temperature (SST) are prescribed to explore the climate of a CONTROL case and the response to variation of the latitudinal profile of SST and to localised and global-scale tropical SST anomalies. A particular focus is the organisation of rainfall over tropical oceans. The idealised configuration is designed to expose differences in the circulation simulated by different models.

Corresponding author: Michael Blackburn, NCAS-Climate, Department of Meteorology, University of Reading, PO Box 243, Earley Gate, Reading, RG6 6BB, UK

E-mail: m.blackburn@reading.ac.uk

C 2013, Meteorological Society of Japan
APE is intended to be one component of a modelling hierarchy - a hierarchy of increasing complexity, of the models themselves and of the experimental configuration to which they are applied. The hierarchy has two distinct but overlapping roles. On the one hand it provides a suite of benchmark experiments to test complex models and their components. This can be considered an evaluation framework. On the other hand it provides a link between theory, observation and complex models in meteorology, the need for which has been stressed by Hoskins (1983) and Held (2005). This can be considered a conceptual framework. Without the full range of models, from the simple 'back-ofthe-envelope' models to the complex, state-of-the-art, computer intensive models, theory tends to proceed with little contact with data from the real atmosphere and results from complex models. In consequence, the framework for interpreting observations and the results from complex models, and also for improving the 
complex models themselves, can become fossilised. Held articulated the need for a modelling hierarchy to link the twin goals of simulation and understanding. There is a degree of overlap between the evaluation and conceptual frameworks, and we will argue that APE has the potential to fulfil a critical role at this interface.

Before describing the APE proposal in section 4, it is first justified by providing some historical, theoretical and modern modelling context in sections 2 and 3 . Future development and use of APE within the modelling hierarchy is discussed in section 5 .

\section{Historical and Theoretical Context}

The earliest theories of atmospheric circulation, by Halley in the 17th century and Hadley in the 18th century, were no doubt influenced in their focus on the Trade winds and the tropical convergence regions by the maritime interests of their country during those times. Their discussion and that of Dove in the 19th century was in terms of a planet on which the zonal asymmetries and continents were of secondary importance. The zonally averaged motion remained the framework for the majority of the 20th century and in particular in the superb monograph of Lorenz (1967) which summarised the contribution of Halley, Hadley and many others. The zonally averaged state and eddy fluxes were at that time synonymous with the general circulation of the atmosphere. Zonal asymmetries per se were not high on the agenda and their impact was through eddy terms.

One episode soon after Hadley's work is particularly relevant in the present context. As discussed by Egger and Pelkowski (2008), in 1746 the Academy of Prussia made the subject of its annual prize the determination of the order and the law which winds would have to observe if the Earth were surrounded everywhere by an ocean, so as to find at all times the direction and the velocity of the wind for every place. The responses to the competition are perhaps of limited relevance to the APE proposal considered here, because the Academy intended a solution based on the theory of tides and specifically excluded the effects of radiative heating. Nevertheless, the motivation, of better understanding through considering a simplified problem, mirrors the motivation for APE described in the introduction. Egger and Pelkowski consider the responses published by the Academy to be the first mathematical models in dynamic meteorology.

Theories of the Hadley circulation have advanced in recent decades, beginning with a numerical model by Schneider (1977) and an elegant analytic model by
Held and Hou (1980). In this theory the Hadley Cell exists when the thermal state in moist equilibrium with the underlying zonal SSTs has a thermal wind that is inconsistent with angular momentum conservation from rest at the equator. The Hadley Cell then exists and acts to reduce the interior temperature gradient below that implied by the SSTs. According to the model this occurs only if the tropical profile with latitude is steeper than quartic. Later, Lindzen and Hou (1988) showed sensitivity of the relative amplitudes of the Cells in the two hemispheres to the SST maximum being displaced from the equator. Displacement of the solar heating by as little as $2^{\circ}$ produces a much stronger circulation than the equatorial case, with a dominant Cell crossing the equator into the winter hemisphere, more consistent with the observed seasonal cycle and intensity of the annual mean circulation. Realism may be further enhanced by including a simple representation of latent heating by convection, either heuristically (Held and Hou, 1980) or by numerical solution (Schneider 1977). In this "moist" Hadley Cell model, broad-scale descent is similar to that in the dry solution but ascent becomes stronger and confined in a narrow region, more like the observed inter-tropical convergence zone (ITCZ).

Alongside this tropic-wide theory, a number of studies have been motivated by the question of whether SST or a dynamical mechanism primarily determines the location of the ITCZ, based on observational evidence that it remains off the equator even in regions with an equatorial SST maximum. A simple thermodynamic argument predicts that the ITCZ and its boundary layer convergence will be coincident with the latitude of maximum SST: ubiquitous deep convection produces the warmest air in depth here, with low pressure at the surface and high pressure aloft driving the horizontal branches of a thermally direct meridional overturning circulation. Dynamical processes may then give a preference for convergence on or off the equator. This leads to the possibility that the ITCZ will be located above a strongly peaked equatorial SST but move off the equator for a weaker tropical SST gradient, with a double ITCZ for weakly peaked SSTs that are symmetric about the equator or, as observed in the eastern Pacific, in the presence of an equatorial minimum in SST. The first dynamical theory, by Charney (1971), applied the concept of conditional instability of the second kind (CISK) to the zonally symmetric tropical flow. In this model, frictionally induced convergence increases with latitude in the cyclonic shear of the easterly trade winds, counteracting a decreasing efficiency of moisture conver- 
gence with decreasing SST away from the equator. The resulting tension between SST and the Coriolis force in this CISK model results in a preferred latitude for the ITCZ. Waliser and Somerville (1994) and Tomas et al. (1999) used momentum balance considerations to suggest that, close to the equator (within about a Rossby radius), low level meridional inflow to an ITCZ is determined frictionally rather than geostrophically, allowing enhanced convergence and feedback with mid-level heating for an ITCZ located around $4-12^{\circ}$ from the equator. However, these studies generally impose part of the solution, either the latitudinal structure of the heating or the pressure field, so it can be argued that they are primarily diagnostic consistency arguments. Other latitudinally varying influences on the ITCZ have been proposed, including the occurrence of inertia gravity waves and friction (e.g. Chao and Chen 2004).

It was recognised early in the development of these theories that ITCZ convection is far from zonally symmetric. Charney's CISK model implies that ITCZ structure is determined by the $2 \mathrm{D}$ zonally symmetric dynamics, and that this then determines the dominant tropical disturbances (Charney 1971, 1974). Conversely, Holton et al. (1971) and Holton (1974) used theory to suggest that the disturbances should set the ITCZ structure.

Zonally asymmetric circulations have since been increasingly studied. The response to tropical heating in a longitudinally confined region, perhaps associated with an SST anomaly there, was analysed by Gill (1980) in terms of a specified deep vertical structure and the equatorial wave modes first discussed by Matsuno (1966). The interpretation of the observed response to such forcing in terms of equatorial Rossby waves to the west and a Kelvin wave to the east has since become a fundamental concept. Hoskins and Karoly (1981) showed that such a tropical response can initiate a stationary Rossby wave that propagates in an almost great circle path to influence higher latitudes. The same equatorial waves can exist as free modes in a dry atmosphere and many studies (e.g. Wheeler and Kiladis 1999; Yang et al. 2007) have looked at the relevance of equatorial waves and other processes to observed organisation of tropical convection.

Much of the research on extra-tropical storm-tracks has also been done in the context of a longitudinally symmetric basic state modelled on that over the ocean basins. In particular, this was the case for the theoretical models of baroclinic instability of Charney (1947) and Eady (1949), and the baroclinic wave life-cycle experiments of Simmons and Hoskins (1978).
Early in the 20th century, Jeffreys (1926) recognised the role of eddies in maintaining both the trade winds and the mid-latitude westerlies against friction through a poleward transport of angular momentum. The westerly momentum sink in the regions of the subtropical jets due to the eddies is important in the angular momentum budget of the Hadley Cells, reducing the jet speeds far below those implied by angular momentum conservation. Recently, Shutts (2006) has emphasised the sensitivity of the Hadley Cell circulations in equatorial $\beta$-plane simulations to the magnitude of momentum sink imposed in his model. Lorenz (1955) quantified both the thermal and mechanical impacts of eddies on the zonally averaged flow in his global energy cycle of Available Potential Energy and Kinetic Energy.

Later, recognition of the conditions under which eddies do and do not influence the zonally averaged flow, by Eliassen and Palm (1961) and Charney and Drazin (1961), led to development of a generalised wave mean-flow interaction theory by Andrews and McIntyre (1976) and Boyd (1976), in which the total forcing of the zonally averaged flow by eddies is characterised by the convergence of a flux of wave activity in the meridional plane, the Eliassen-Palm or EP flux. This combination of the eddy heat and momentum fluxes, together with a residual meridional circulation, then describes the forcing and evolution of the zonally averaged state in a Transformed Eulerian Mean framework (Edmon et al. 1980). This theoretical and diagnostic framework remains ideally suited to idealised modelling studies with zonally symmetric boundary conditions and forcing.

\section{Modern Modelling Context}

\subsection{The conceptual role of aqua-planet configura- tions}

Many of the theoretical studies discussed so far used numerical models, including AGCMs, to test the theory and explore the expected behaviour in comparison with observations. By the nature of the theories being tested, many of these were aqua-planet models. In fact many early numerical studies were conceived as aquaplanet models, despite their representation of moist processes being so simplified that interaction with an underlying ocean was implicit rather than directly modelled. Phillips' (1956) pioneering experiment to investigate the growth of mid-latitude baroclinic disturbances and their role in maintaining the zonally averaged flow used a 2-layer quasi-geostrophic $\beta$ plane model with zonally symmetric boundary conditions and forcing. Bates (1970) used the same dynam- 


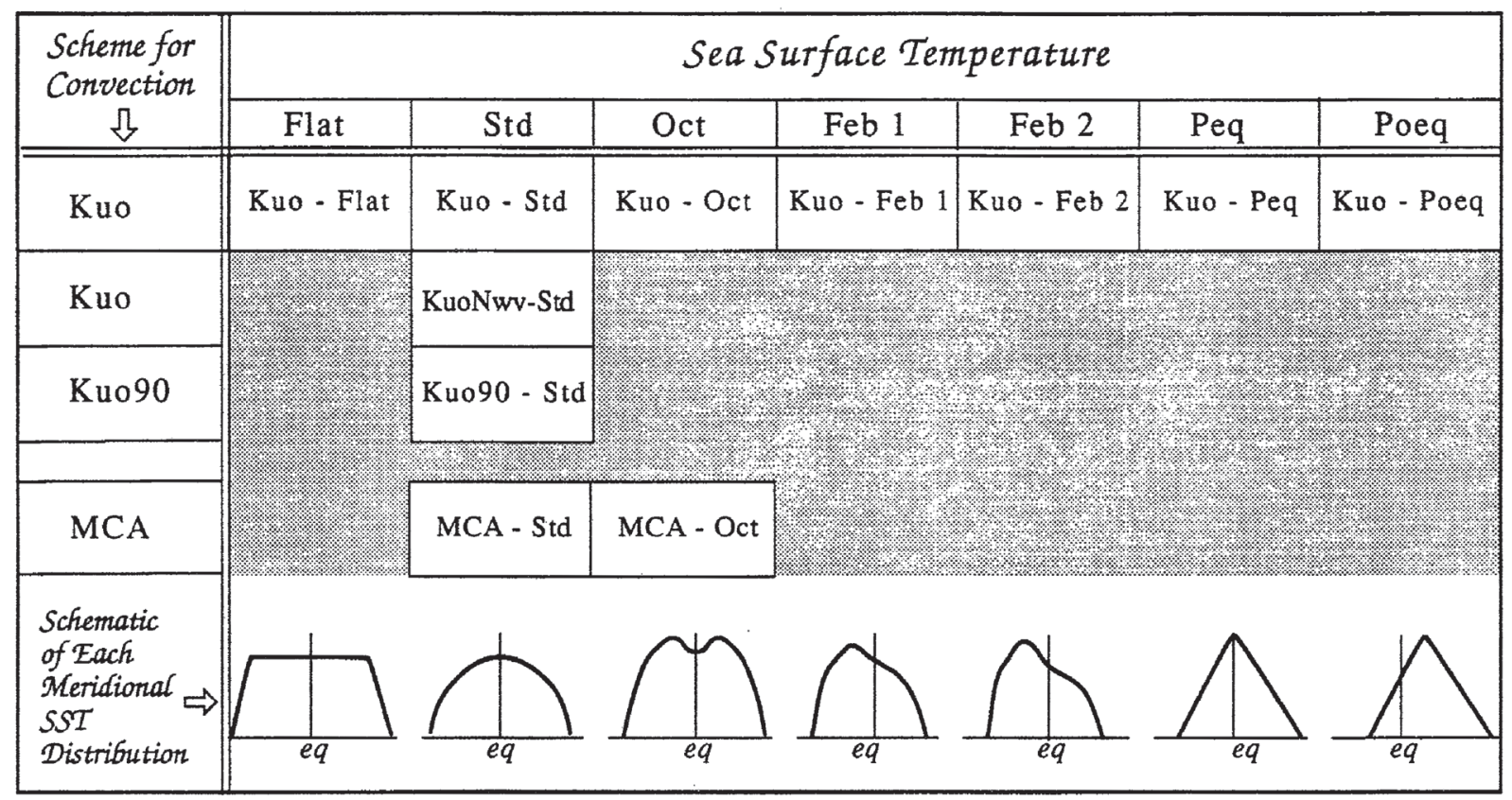

Fig. 1. Aqua-planet experiments and SST profiles used by Hess et al. (1993). Copyright 1993 American Meteorological Society.

ical formulation with Charney's CISK representation of tropical convection to study a zonally symmetric ITCZ and the nature of disturbances on it. The model produced a strong ITCZ at $14^{\circ}$ latitude, despite a reference temperature with an equatorial maximum and a quadratic profile with latitude, which Held and Hou's later theory predicts should produce a Hadley Cell with equatorial ascent. This was perhaps an early indication of the dependence of simulated tropical behaviour on modelling choices, particularly that of convective parameterization, found in later modelling studies.

Hayashi and Sumi (1986) were the first to use a full primitive equation AGCM in an idealised study on a water-covered Earth, introducing the term "aquaplanet" to describe the configuration. They found spontaneous organisation of equatorial convection into slow eastward propagating "super-clusters" that were modulated on the planetary scale in a similar way to the observed 30-60 day intraseasonal oscillation. Their model also produced a double ITCZ with a rainfall minimum on the equator, even with an equatorial maximum of SST. This seminal work spawned an increasing number of idealised modelling studies of tropical convection that have continued to today.

Sumi (1992), using an even simpler AGCM configuration of globally uniform SST and radiative cooling, still found spontaneous organisation of tropical convection. Tropical rainfall was banded with a single or double maximum about the equator, but much weaker than the ITCZ obtained with a more realistic SST profile. Sumi concluded that convective organisation is dependent on equatorial wave dynamics, and therefore on planetary rotation rate. Hess et al. (1993) used a number of idealised zonally symmetric SST profiles, reproduced in Fig.1, ranging from one with an equatorial minimum through flat in the tropics to sharply peaked, with SST symmetric or asymmetric about the equator. Different convective parameterizations in the same model were found to produce qualitatively different tropical circulations, both for the mean flow and transients for a standard SST case, and different sensitivities to SST profile. Hess et al. concluded that GCM simulations are likely to be sensitive to parameterization in tropical regions which have relatively weak SST gradients, something that has been borne out in subsequent studies. However, simulations have proved to be sensitive to many model processes and choices. For example, Numaguti (1993) and Chao and Chen (2004) obtained a single or double ITCZ by disabling specific physical interactions in their models, including the dependence of surface evaporation on wind speed, and Williamson and Olson (2003) found a marked sensitivity to time step in the NCAR model.

At the same time, coupled atmosphere-ocean 
GCM simulations of present-day Earth climate have persistently exhibited an unrealistic double ITCZ in the tropical Pacific (Mechoso et al. 1995; Lin 2007). Such a structure is observed in boreal Spring in the east Pacific, but models generally exaggerate and extend the equatorial SST cold-tongue and off-equator deep convection across the Pacific basin in all seasons. Lin (2007) compared coupled and prescribed-SST simulations from phase 3 of the Coupled Model Intercomparison Project (CMIP3, Meehl et al. 2007). Consistent with an earlier analysis of two coupled models by Schneider (2002), Lin deduced that the double-ITCZ arises through ocean-atmosphere coupling, but originates in biased precipitation and circulation responses to realistic SSTs that are intrinsic to the uncoupled AGCMs. In the CMIP3 AGCMs, excessive tropical precipitation and Pacific trade winds lead to biased surface stress and surface heat flux. This generates excessive equatorial upwelling and SST errors in the coupled models, giving rise to a feedback that splits the ITCZ.

Aqua-planet simulations could be used to investigate the underlying AGCM precipitation biases, by characterising ITCZ behaviour in a controlled context, for a range of idealised zonally symmetric and zonally varying SSTs. This would also provide a link to the conceptual models discussed earlier, which predict splitting of a single equatorial ITCZ as the tropical SST profile flattens. The surface stress associated with easterly trade winds implies that coupling to an ocean could destabilise an equatorial ITCZ, with excessive trades exaggerating the effect. Differences in ITCZ splitting and latitudinal profiles of zonal wind stress produced by different AGCMs could be used to compute implied variations in equatorial upwelling, and these could be compared to the upwelling produced when each aquaplanet AGCM is coupled to a dynamical tropical ocean.

Aqua-planet AGCMs have also been used to study aspects of the mid-latitude storm tracks, including the behaviour of baroclinic wave packets (Lee and Held 1993), wave breaking (Lee and Feldstein 1996) and variability of the westerly jet (Feldstein and Lee 1996). Idealised modelling of annular variability has received increasing attention, including further studies using aqua-planets (e.g. Cash et al. 2002). Aqua-planets have been used to study sensitivity of idealised storm tracks to the underlying latitudinal SST structure (Brayshaw et al. 2008, Lu et al. 2010).

With increasing computer power, Cloud System Resolving Models (CSRMs) have recently been applied to large aqua-planet domains, generally non-rotating with constant SST, to investigate the sponta- neous organisation of convection in radiative convective equilibrium (Bretherton et al. 2005; Stephens et al. 2008). These studies have found a "self-aggregation" of deep convection into one or more clusters that depends on cloud radiative and/or surface flux feedbacks. In the work mentioned above, Shutts (2006) used a form of CSRM on an equatorial $\beta$-plane aquaplanet with varying SST to investigate the large scale tropical circulation.

\subsection{The evaluation role of aqua-planet configura- tions}

It is clear from the preceding discussion that aquaplanet configurations have provided both a simplified context to understand more complex observed and modelled behaviour and a link to theory and simpler models. This is the conceptual role of the modelling hierarchy discussed earlier. However, a number of such studies also highlight a dependence of the simulated circulation, particularly in the tropics, on modelling choices. Moreover, the variety of GCM behaviour seen in realistic simulations appears more starkly in aquaplanet simulations. This suggests an evaluation role for aqua-planet configurations in the AGCM modelling hierarchy.

The present hierarchy of AGCMs includes at the simpler end baroclinic models of varying complexity. A further simplification which retains prognostic divergent motion is the shallow water equations. A benchmark suite of test cases has been developed to evaluate different numerical formulations of the nonlinear shallow water equations on the sphere (Williamson et al. 1992). Baroclinic dynamical cores have been used with only internal diffusion for baroclinic lifecycle simulations (Simmons and Hoskins 1978) and for simulating the direct impact of tropical heating (Hoskins and Karoly 1981). Deterministic initial-value test cases of baroclinic instability have been proposed for these primitive equations models (Polvani et al. 2004; Jablonowski and Williamson 2006). Following James and Gray (1986) and Held and Suarez (1994), long-period experiments have been performed in which temperature is relaxed towards a prescribed 2-D field, with the Held-Suarez configuration designed as a further benchmark test case. A related test was proposed by Boer and Denis (1997), in which the thermodynamic forcing combines a dominant specified time-independent heating function with a weak linear relaxation towards a specified zonal climatology.

The Newtonian-relaxed dynamical cores have proved extremely useful in many experiments aimed at understanding basic aspects of observed atmospheric 


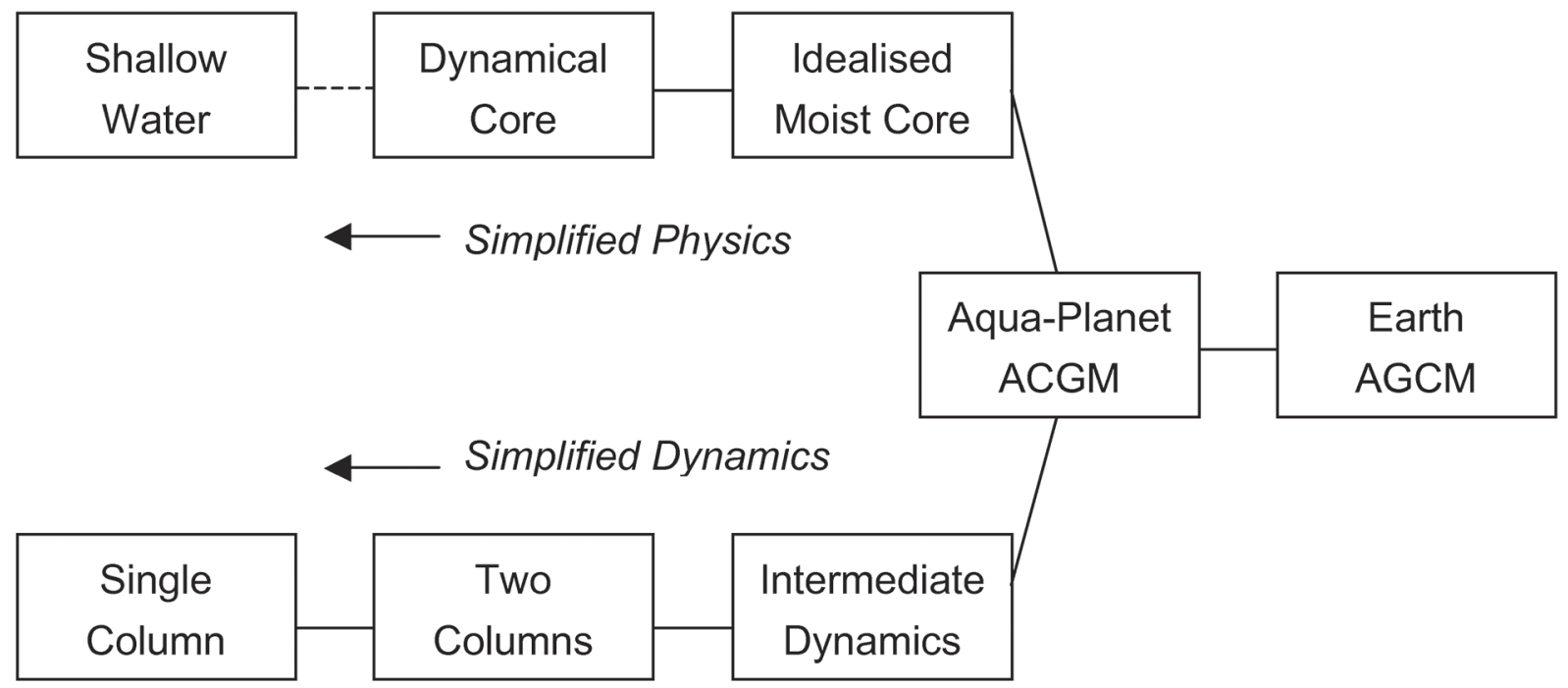

Fig. 2. A modelling hierarchy for AGCM evaluation.

structure and variability. Following James and Gray (1986), this configuration with zonally symmetric forcing has been used extensively to study low frequency variability of the zonally averaged flow and baroclinic disturbances (e.g. Robinson 1991; James and James 1992, Yu and Hartmann 1993; Akahori and Yoden 1997 and subsequently) and the impact of stratospheric perturbations on the troposphere (e.g. Polvani and Kushner 2002; Kushner and Polvani 2004; Haigh et al. 2005 and subsequently). Dynamical cores have also been used to interpret the sensitivity of AGCMs to numerical formulation, in some cases by showing the importance of an explicit representation of moist processes in such sensitivities. For example, the introduction of semi-Lagrangian dynamical cores corrected or significantly reduced the polar tropopause cold bias seen historically in AGCM climate simulations, but this impact was not seen when the same models were applied to the Held-Suarez test case (Chen and Bates 1996; Chen et al. 1997; Williamson and Olson 1998; Williamson et al. 1998). Clearly the different responses are associated with the very different parameterizations of physical processes.

At the complex end of the hierarchy, next to Earth System Models and Coupled Ocean-Atmosphere GCMs, AGCMs with their full package of physical parameterizations are used with prescribed SSTs. This configuration has proved stimulating in consideration of the response to realistic SST and sea-ice anomalies, and has been used widely in model intercomparisons such as the Atmospheric Model Intercompar- ison Project (AMIP, Gates 1992; Gates et al. 1999). However, often the interpretation of the experiments has proved difficult and firm conclusions frustratingly elusive because of the complexity of the simulations.

There is therefore a large gap in the hierarchy between, on the one hand, atmospheric dynamical cores with Newtonian relaxation and idealised configurations and, on the other hand, the AGCMs with their dynamical cores plus full physics packages and complex geography and prescribed SSTs. In this gap can be placed full physics AGCMs but with much simplified lower boundary conditions, in particular a water-covered Earth with simple SSTs, the aquaplanet configuration introduced by Hayashi and Sumi (1986). Other ways of filling portions of this gap have been tried. Simple moist and radiative physics can be added to baroclinic wave simulations (e.g. Hoskins 1978; Gutowski et al. 1992) or can be used to replace complex parameterization suites of AGCMs in aquaplanet configurations (e.g. Frierson et al. 2006). This latter approach, which we will call an Idealised Moist Core, will be developed further in the discussion in section 5. Numerical weather prediction provides a continuous evaluation of operational models, and has been proposed as a method to evaluate AGCMs used for climate studies (Phillips et al. 2004), as also has seasonal prediction (Palmer et al. 2008).

The preceding range of models and test cases may be considered as one branch of a wider modelling hierarchy used for AGCM evaluation, represented schematically in Fig. 2. With the exception of the shallow 
water model, in this branch the AGCM dynamical core is fixed at its most complex and the representation of physical (as opposed to dynamical) processes increases in complexity. A parallel branch exists in which the complex physical parameterization package is fixed and coupled to a range of dynamical models. The simplest such configuration is a single column model (SCM), used widely to test AGCM parameterizations. However, the large scale dynamics is prescribed in an SCM, with very limited possibility for interaction between the physics and dynamics to expose feedbacks and sensitivities. More complex formulations couple an SCM to a reference column (Sobel and Bretherton 2000), or couple two SCMs with simplified dynamics (e.g. Nilsson and Emanuel 1999; Raymond and Zeng 2000). Beyond this, a range of intermediate dynamics and configurations is possible, up to use of the complete AGCM in idealised configurations such as aqua-planets and finally realistic configurations. The aqua-planet is notable for being the simplest experimental configuration in which the complete dynamical and physical parameterization components of an AGCM are coupled together.

\section{APE Proposal and Aims}

The foregoing review emphasises the need for a systematic analysis of AGCM behaviour, on both conceptual and evaluation grounds, to improve our understanding and simulation of key components of the atmospheric circulation. Aqua-planet configurations have already proved useful in this regard, highlighting specific atmospheric phenomena, providing a link with conceptual models of those phenomena, and bridging a gap between complete AGCMs in realistic configurations and simpler models in both branches of the existing modelling hierarchy. A benchmark experiment or suite of experiments has been devised for each existing member of the hierarchy and the AquaPlanet Experiment (APE) was proposed by Neale and Hoskins (2000a) to fulfil that role for the aqua-planet configuration.

\subsection{The Proposal}

Neale and Hoskins proposed a suite of eight simulations, each comprising an idealised distribution of SST and forced by perpetual equinoctial insolation. The SSTs are defined in the Appendix for completeness. Five SSTs, shown in Fig.3, are zonally symmetric profiles and the first 4 of these are also symmetric about the equator. They have varying curvature in the tropics, from a PEAKED profile in which the mid-latitude SST gradient continues to the equator, through a

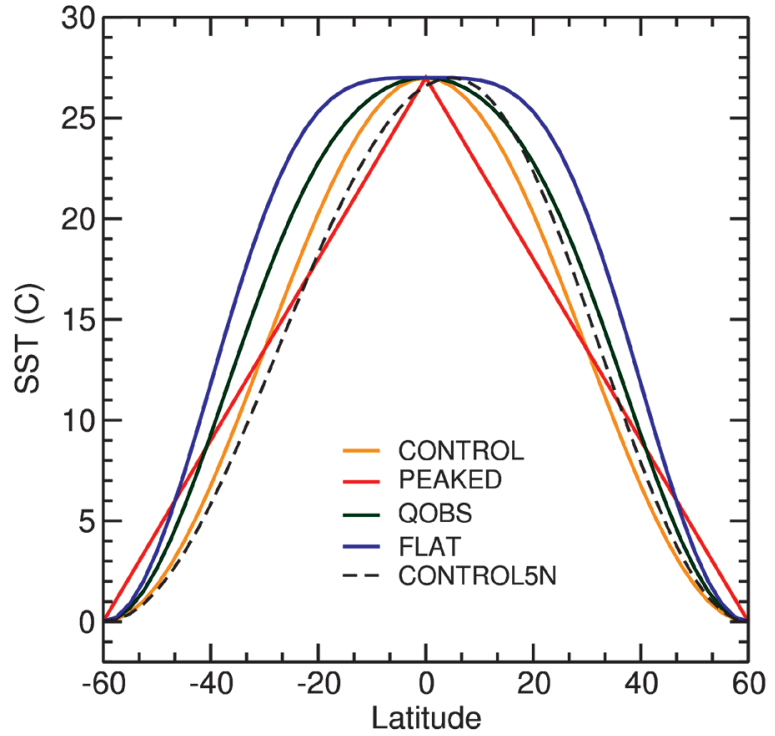

Fig. 3. Five zonally symmetric sea surface temperature profiles prescribed in APE.

CONTROL with $\sin ^{2}(\phi)$ variation and QOBS which is closer to the observed profile in the Pacific, to a $\sin ^{4}(\phi)$ profile, denoted FLAT, which is the limiting thermodynamic profile for the existence of a Hadley circulation in the Held and Hou model. The fifth profile, CONTROL_5N, is asymmetric about the equator, moving the maximum SST in the CONTROL case to $5^{\circ} \mathrm{N}$. These zonally symmetric profiles systematise those used by Hess et al. (1993), and explicitly test the relevance of the theoretical limit of the Held and Hou model to AGCMs. A further three SST distributions, shown in Fig.4, add tropical anomalies to the CONTROL profile. 1KEQ and 3KEQ add a localised equatorial warm anomaly of $1 \mathrm{~K}$ and $3 \mathrm{~K}$ respectively, while $3 \mathrm{KW} 1$ adds a global scale anomaly of $3 \mathrm{~K}$ amplitude with wavenumber one variation in longitude.

The experimental design has been further developed to ensure as far as possible a precise configuration for the systematic comparison of AGCMs. This is summarised in the Appendix and is described in detail in Williamson et al. (2012). In addition to the eight SST distributions, the design defines the insolation and a zonally symmetric ozone climatology, both of which are precisely symmetric about the equator, plus the concentrations of well-mixed greenhouse gases, aerosols and the geophysical constants. All simulations should be spun-up to equilibrium for at least 6 months, before an experimental period of 3 years. With perpetual equinox insolation, this was found to 

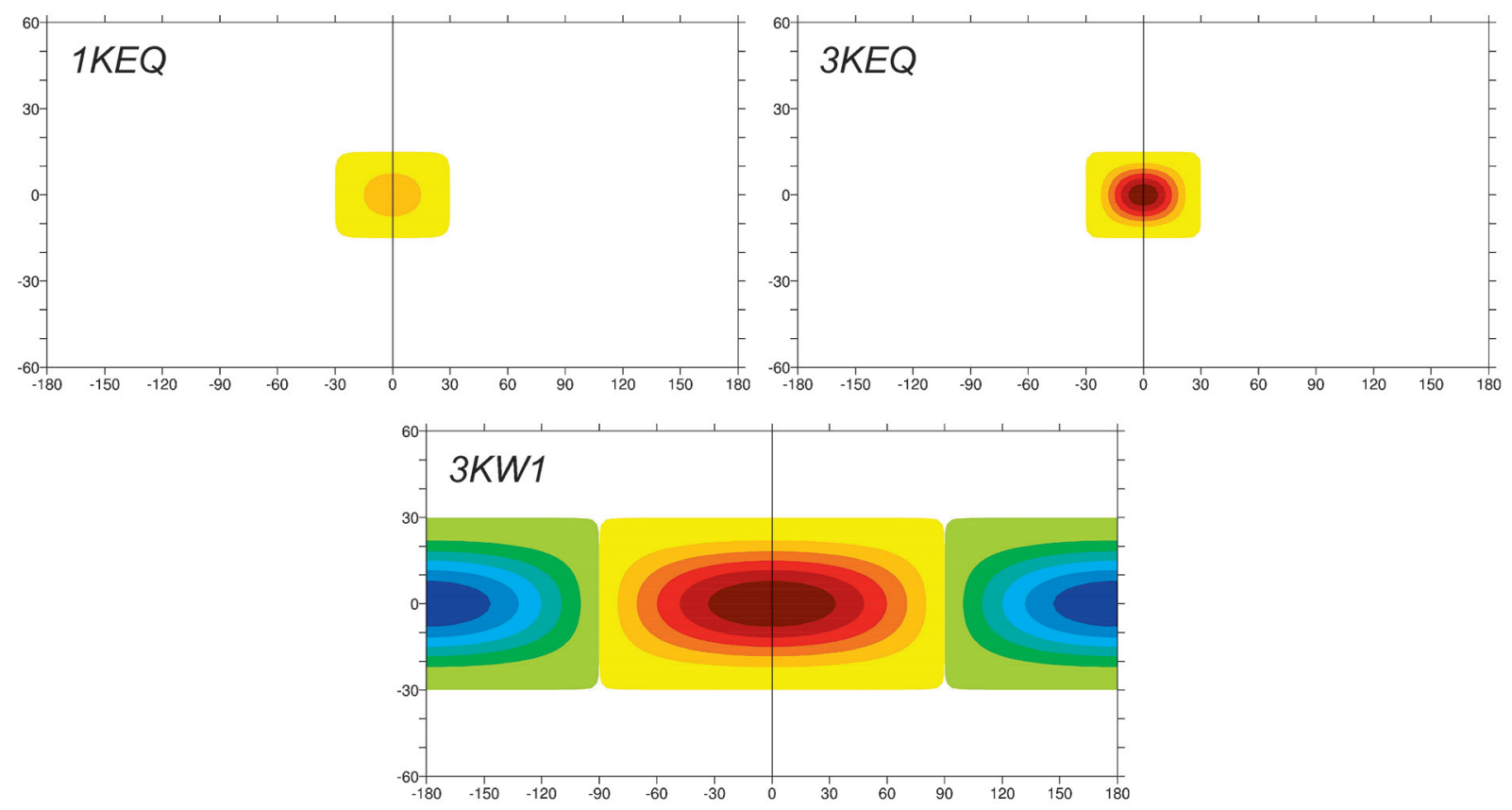

Fig. 4. Three tropical sea surface temperature anomalies added to the APE CONTROL case. Shading interval $0.5^{\circ} \mathrm{C}$.

be adequate to achieve convergence of many climate statistics.

\subsection{Aims}

A general justification has already been given for APE as a component of the modelling hierarchy. The specific aims of the experiment can be expressed in terms of the evaluation and conceptual roles of the hierarchy.

\section{Evaluation Aims}

- APE is intended to be an idealised benchmark experiment for AGCMs.

- The idealised configuration is designed to expose differences in the circulation simulated by different models. In particular, forcing of the circulation is zonally symmetric in five of the eight experiments, apart from inclusion of the diurnal cycle of insolation. All eddying motion must then arise spontaneously, either by dynamical instability or by interaction of the resolved fluid dynamics and parameterized physical processes.

- APE aims to stimulate research to understand the causes of inter-model differences, arising from different subgrid-scale parameterization suites, different dynamical cores, and different methods of coupling the two.

\section{Conceptual Aims}

- The simplified configuration focuses attention on the distribution and variability of convection in the tropics and on the storm-tracks in mid-latitudes.

- Phenomena are cleanly separated into a zonally averaged state and eddies. This aids interpretation, by allowing established diagnostic frameworks to be applied and links to be made to simpler models and theory.

- The symmetric SST experiments can be repeated in a two-dimensional framework, in the absence of zonal asymmetries. The resulting circulation relates more closely to conceptual models of the Hadley circulation and ITCZ discussed in section 2.

- The experiments can also be performed in more idealised models, allowing clean comparison with complex AGCMs and, at the same time, closer links to conceptual models.

\subsection{Specific Goals}

Not all the goals listed below are explored in the initial coordinated model intercomparison, reported in other papers in this Special Issue. But all are longer term goals for APE.

- Quantify the variation among models of key components of atmospheric circulation, and compare this modelling uncertainty with that in SST-forced Earth-climate (AMIP) simulations by the same 
models.

- Explore AGCM simulation of the expected breakdown of the Hadley circulation and equatorial ITCZ as the SST profile is flattened in the tropics.

- Compare the response of the tropical circulation to displacement of the SST maximum into a "summer" hemisphere with conceptual models. These predict an intensification of an asymmetric Hadley circulation into the "winter" hemisphere.

- Compare AGCM simulations of tropical variability and its projection onto the spectrum of equatorially trapped waves. Unlike the extra-tropics, where dynamical instability is the dominant source of eddy variability in the aqua-planet configuration, tropical variability arises from interaction of the dynamics and physical (moist, radiative) processes that must be parameterized in AGCMs. Tropical variability is therefore expected to vary more widely among models.

- Determine the extra-tropical response to changes in SST profile, and how these are related to changes in the tropical circulation.

- Determine the circulation response to a localised anomaly in tropical SST, what processes determine the local and global responses, and how these vary between models.

- Determine the circulation response to a planetary scale anomaly in tropical SST. This involves the generation and propagation of planetary-scale Rossby waves, their longitudinal modulation of the extra-tropical storm-track and their impact on meridional transports.

A number of these aspects were investigated for a single AGCM by Neale and Hoskins (2000b). A coordinated model intercomparison will reveal the range of behaviour and circulation responses in a number of models. Sensitivity studies in individual models will reveal dependences on specific modelling choices.

\section{Discussion}

Context has been provided for the Aqua-Planet Experiment, first proposed as a benchmark for AGCMs on a water-covered Earth by Neale and Hoskins (2000a). The experiment and its aims have been described. APE bridges a gap in the existing modelling hierarchy that is used to evaluate complex models and to provide a link between realistic simulation and conceptual models of atmospheric phenomena. The simplified aqua-planet configuration is designed to expose differences between models due to the interaction of modelled physics and dynamics and to focus attention on particular phenomena and their response to changes in the underlying distribution of sea surface temperature.

Other papers in this Special Issue describe a first coordinated intercomparison of AGCM simulations using the APE configuration. Certain aspects, including the mid-latitude storm track and its response to changes in SST and tropical circulation, remain to be explored in depth.

APE is intended to be used and developed in a number of ways, covering the various contexts described in this paper. This first APE intercomparsion is a benchmark of current model behaviour and is intended to stimulate ongoing evaluation of AGCMs using the APE configuration. In the immediate future, one of the APE experiments, QOBS, is included in CMIP5 (Taylor et al. 2009), alongside the AMIP benchmark and coupled ocean-atmosphere experiments.

In contrast to realistic simulation in weather prediction and climate, there is no verification for APE to determine the fidelity and accuracy of individual models. APE simulations may therefore be best evaluated in conjunction with parallel AMIP simulations, which also use prescribed SSTs but which can be evaluated against observations. The same argument can be applied to experiments for simpler models in the hierarchy, including the Held-Suarez dynamical core benchmark experiment, for which there is no reference solution or independent knowledge of "truth". It is instructive to consider what might constitute a reference solution for APE or the dynamical core. One approach is investigation of the possible convergence of model climate with increasing resolution. If multiple models were to converge towards a unique solution for a given benchmark experiment, and there is no guarantee that they would, there might be some confidence that this was indeed the "true" climate. In a dynamical core, the climate forcing is specified analytically, with parameter settings independent of resolution, but the explicit numerical damping is tuned for each resolution, in a way that depends on each model's numerical scheme. As a result, convergence of multiple models towards a unique climate is not guaranteed. However, in APE the tuning and even choice of physical parameterization schemes is resolution dependent. This is particularly true of convection schemes, which must either be switched off or be designed to switch off gradually as convective scale motions become resolved. Such resolution dependence renders the possibility of convergence of multiple moist AGCMs towards a unique climate significantly less likely than for dynamical cores. However, the approach of increasing model 
resolution could, at the least, reduce modelling uncertainty.

Further ahead, it may be possible to use global CSRMs and then Cloud Resolving Models (CRMs) to simulate the equilibrated APE climate. Such fine scale models have already proved useful for evaluating AGCM parameterizations in observed and idealised case studies, so it is possible that multiple CRMs may show a higher degree of convergence than AGCMs, thereby further reducing uncertainty as to the target in APE. These issues are discussed in the light of the AGCM simulations of the APE CONTROL case by Blackburn et al. (2013).

APE may also be used to investigate internal atmospheric variability and the response to external forcing in an idealised context. This has arguably been the main use of the Held-Suarez configuration since its introduction in 1994, with numerous studies perturbing the Newtonian reference state and investigating the resulting changes in mean state and variability. In contrast to Held-Suarez, the idealised APE climate includes realistic moist and radiative feedbacks to any imposed forcing. As an example, Kodama and Iwasaki (2009) perturbed the APE CONTROL SSTs globally and regionally to investigate aspects of the atmospheric response to increased greenhouse gases. CMIP5 includes a global $+4 \mathrm{~K}$ SST perturbation and a $4 \mathrm{xCO}_{2}$ version of the APE QOBS experiment, allowing components of the climate change response to be investigated in a simplified framework.

APE is also a basis for more complex configurations to understand aspects of global circulation. For example Brayshaw et al. (2009) and Saulière et al. (2012) have added idealised continents and SST anomalies to investigate the "building blocks" of the observed north Atlantic and north Pacific storm-track climate respectively. Xie and Saiki (1999) looked at summer monsoon development when an idealised Asia is added. Idealised geographical configurations could also have an evaluation role, particularly to investigate the land-sea distribution of tropical precipitation in different AGCMs without resorting to realistic geography.

Further development of the modelling hierarchy has already been discussed. An additional possibility is simplification of APE, since each AGCM retains its unique dynamical core and suite of parameterizations in APE, which makes it difficult to attribute differences in model behaviour to particular modelling choices. Studies in individual models help to elucidate such sensitivities, while simplification of model processes in multiple models reduces differences between their formulations. Simpler models also relate more closely to conceptual models of particular phenomena. Options for simplifying the dynamics, while retaining the AGCM parameterizations, are probably limited, beyond imposing zonal symmetry. Of the parameterizations, radiative processes could be simplified by switching off the radiative effects of cloud, or by using a single water vapour climatology or grey body approximation for clear-sky radiation, or even by using a prescribed cooling. These simplifications would constrain forcing of convection and turbulence in different models. Ultimately, a single simplified parameterization suite could be used in all AGCMs, what might be called an Idealised Moist Core. This would be a moist equivalent of the Held-Suarez configuration, and could be used to explore atmospheric phenomena and sensitivities to model numerics in the presence of switchable moist feedbacks. The simplified model of Frierson et al. (2006) could be seen as one such model.

Discussion of the modelling hierarchy has so far been restricted to atmosphere-only models. However, prescribing insolation and SST in an AGCM simulation such as APE or AMIP does not in general produce an internally consistent circulation. The planet is not in global energy balance, since the prescribed SST acts as an infinite heat source or sink, and the resulting atmospheric circulation is not constrained to be consistent with the ocean circulation that is implied by spatial variations of the net surface energy flux (Held 2001). To obtain an internally consistent idealised climate, Lee et al. (2008) proposed an aqua-planet benchmark experiment in which the AGCM is coupled to a global mixed layer ocean. The resulting equilibrated climate includes internally determined SSTs in the absence of oceanic transport. Kang et al. (2008) imposed an inter-hemispheric ocean heat transport on this configuration, to study the response of the ITCZ to extratropical thermal forcing. Experimental configurations such as these allow climate sensitivity to be studied in an energetically consistent model, but the ocean-atmosphere coupling increases the diversity of model responses. Coupled and uncoupled APE configurations therefore have distinct uses, highlighting the importance of varying complexity in the modelling hierarchy.

It is hoped that the APE experiments, analyses and comparisons described in the papers that follow in this Special Issue will encourage those in the community who develop and modify AGCMs to use this framework as a standard evaluation component in their future research. 


\section{Acknowledgments}

It is a pleasure to acknowledge David Williamson for help in developing the experimental design for APE and for his comments on the manuscript. We also thank Shigeo Yoden and an anonymous reviewer for their comments, which helped to significantly improve the manuscript.

\section{Appendix Experimental design}

The experimental design for APE is summarised here for completeness. It is fully specified in Williamson et al. (2012) using the SST distributions originally defined by Neale and Hoskins (2000a). A recommended set of standard diagnostics to be collected from each experiment is also specified in Williamson et al. (2012). The experiment is specified in a similar manner to the second phase of AMIP, using a set of requirements and recommendations.

\section{Requirements}

1. Surface Boundary Conditions.

SST is prescribed for the eight experiments according to formulae in Box 1. The five zonally symmetric profiles and three tropical anomalies are shown in Fig. 3 and Fig.4. There is no sea ice (minimum SST is $0^{\circ} \mathrm{C}$ ). 2. Radiative forcing and orbital parameters.

Fixed equinoctial insolation, symmetric about the equator but including the diurnal cycle, is prescribed in all experiments. The solar constant is $1365 \mathrm{~W} \mathrm{~m}^{-2}$. This is achieved by modifying the Earth orbit parameters, setting eccentricity and obliquity to zero, to give a circular equinoctial orbit. The distribution of solar irradiance is then independent of the calendar.

3. Well-mixed radiatively active gases.

$\mathrm{CO}_{2}$ mixing ratio is 348 ppmv, as in AMIP II. 4. Ozone.

A zonally symmetric latitude-height distribution of ozone is specified, symmetrised about the equator, corresponding to the annual mean climatology used in AMIP II. The data are available from the APE website (http://climate.ncas.ac.uk/ape/).

5. Simulation period.

All simulations should be spun-up to equilibrium for at least 6 months, before an experimental period of 3 years. Each experiment should be started from a model-simulated state, obtained from either a realEarth or previous aqua-planet integration. The 6-month spin-up should be checked to establish that equilibration was achieved during this period.

\section{Recommendations}

1. Recommended values of the geophysical constants and parameters are given in Williamson et al. (2012).

2. Well-mixed radiatively active gas concentrations follow AMIP II recommendations.

$\mathrm{CH}_{4}$ : 1650 ppbv; $\mathrm{N}_{2} \mathrm{O}: 306$ ppbv. Halocarbon concentrations should yield $\sim 0.24 \mathrm{~W} \mathrm{~m}^{-2}$ radiative forcing. Use of an "equivalent" $\mathrm{CO}_{2}$ is not recommended.

3. Aerosols.

There should be no radiatively active aerosol. Any aerosol specification for cloud condensation should use an oceanic distribution which is fixed in time, zonally symmetric and symmetric about the equator.

4. Atmospheric Mass.

The initial dry mass of the atmosphere should be equivalent to a global mean surface pressure of 101080 $\mathrm{Pa}$. This is $101325 \mathrm{~Pa}$ minus $245 \mathrm{~Pa}$, which corresponds to a global moisture content of $25.006 \mathrm{~kg} \mathrm{~m}^{-2}$ using the recommended value for surface gravity. Dry mass should be conserved throughout the integration. There is no topography.

5. Calendar.

A 365 or 360 day year, with variable- or fixed-length months respectively, should be used. The 3.5 year integration length means that a realistic calendar can be used if integrations are started in March of a leap year. Insolation does not follow the calendar.

\section{References}

Akahori, K., and S. Yoden, 1997: Zonal flow vacillation and bimodality of baroclinic eddy life cycles in a simple global circulation model. J. Atmos. Sci., 54, 23492361.

Andrews, D. G., and M. E. McIntyre, 1976: Planetary waves in horizontal and vertical shear: The generalized Eliassen-Palm relation and the mean zonal acceleration. $J$. Atmos. Sci., 33, 2031-2048.

Bates, J. R., 1970: Dynamics of disturbances on the intertropical convergence zone. Quart. J. Roy. Meteor. Soc., 96, 677-701.

Blackburn, M., D. L. Williamson, K. Nakajima, W. Ohfuchi, Y. O. Takahashi, Y.-Y. Hayashi, H. Nakamura, M. Ishiwatari, J. McGregor, H. Borth, V. Wirth, H. Frank, P. Bechtold, N. P. Wedi, H. Tomita, M. Satoh, M. Zhao, I. M. Held, M. J. Suarez, M.-I. Lee, M. Watanabe, M. Kimoto, Y. Liu, Z. Wang, A. Molod, K. Rajendran, A. Kitoh, and R. Stratton, 2013: The Aqua-Planet Experiment (APE): CONTROL SST simulation. J. Meteor. Soc. Japan, 91A, 17-56, doi:10.2151/jmsj.2013-A02.

Boer, G. J., and B. Denis, 1997: Numerical convergence of the dynamics of a GCM. Climate Dyn., 13, 359-374.

Boyd, J. P., 1976: The noninteraction of waves with the 


\section{APE Sea Surface Temperatures}

1. CONTROL:

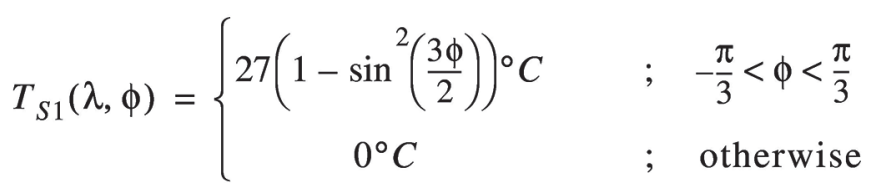

2. PEAKED:

$$
T_{S 2}(\lambda, \phi)=\left\{\begin{array}{cc}
27\left(1-\frac{3|\phi|}{\pi}\right)^{\circ} C & ; \quad-\frac{\pi}{3}<\phi<\frac{\pi}{3} \\
0^{\circ} C & ; \quad \text { otherwise }
\end{array}\right.
$$

3. FLAT:

$$
T_{S 3}(\lambda, \phi)=\left\{\begin{array}{cc}
27\left(1-\sin ^{4}\left(\frac{3 \phi}{2}\right)\right){ }^{\circ} C & ; \quad-\frac{\pi}{3}<\phi<\frac{\pi}{3} \\
0{ }^{\circ} C & ; \quad \text { otherwise }
\end{array}\right.
$$

4. QOBS:

$$
T_{S 4}(\lambda, \phi)=\left(T_{S 1}+T_{S 3}\right) / 2
$$

5. CONTROL-5N:

$$
T_{S 5}(\lambda, \phi)=\left\{\begin{array}{cc}
27\left(1-\sin ^{2}\left(\frac{90}{55}\left[\phi-\frac{\pi}{36}\right]\right)\right){ }^{\circ} C & ; \quad \frac{\pi}{36}<\phi<\frac{\pi}{3} \\
27\left(1-\sin ^{2}\left(\frac{90}{65}\left[\phi-\frac{\pi}{36}\right]\right)\right){ }^{\circ} C & ; \quad-\frac{\pi}{3}<\phi<\frac{\pi}{36} \\
0^{\circ} C & ; \quad \text { otherwise }
\end{array}\right.
$$

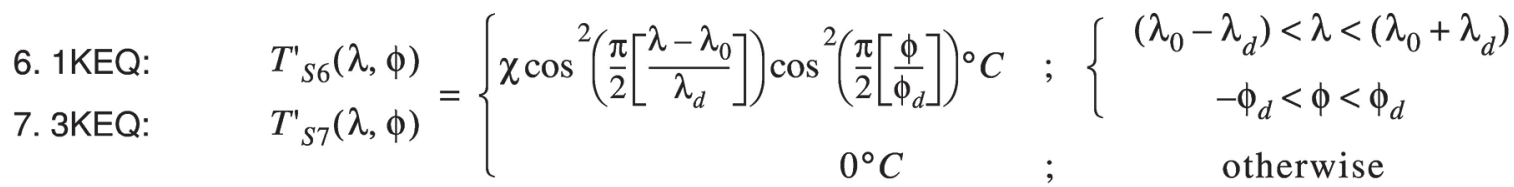

8. 3KW1:

$$
T_{S 8}^{\prime}(\lambda, \phi)=\left\{\begin{array}{cc}
\chi \cos \left(\lambda-\lambda_{0}\right) \cos ^{2}\left(\frac{\pi}{2}\left[\frac{\phi}{\phi_{d}}\right]\right){ }^{\circ} C & ;-\phi_{d}<\phi<\phi_{d} \\
0^{\circ} C & ; \text { otherwise }
\end{array}\right.
$$

$\chi=1^{\circ} \mathrm{C}\left(T_{S 6}^{\prime}\right) ; \chi=3{ }^{\circ} \mathrm{C}\left(T_{S 7}^{\prime} ; T_{S 8}^{\prime}\right)$ : Maximum magnitude of SST anomaly

$\lambda_{0}=0^{\circ} E ; \lambda_{d}=30^{\circ} ; \phi_{d}=15^{\circ}: 1 \mathrm{KEQ}, 3 \mathrm{KEQ}$ : longitude of maximum anomaly; half widths.

$\lambda_{0}=0^{\circ} \mathrm{E} ; \quad \phi_{d}=30^{\circ}: \quad 3 \mathrm{KW} 1$ : longitude of maximum anomaly; half width. 
zonally averaged flow on a spherical earth and the interrelationships on eddy fluxes of energy, heat and momentum. J. Atmos. Sci., 33, 2285-2291.

Brayshaw, D. J., B. J. Hoskins, and M. Blackburn, 2008: The storm-track response to idealized SST perturbations in an aquaplanet GCM. J. Atmos. Sci., 65, 2842-2860.

Brayshaw, D. J., B. J. Hoskins, and M. Blackburn, 2009: The basic ingredients of the north Atlantic storm track. Part I: Land-sea contrast and orography. J. Atmos. Sci., 66, 2539-2558.

Bretherton, C. S., P. N. Blossey, and M. Khairoutdinov, 2005: An energy-balance analysis of deep convective self-aggregation above uniform SST. J. Atmos. Sci., 62, 4273-4292.

Cash, B. A., P. J. Kushner, and G. K. Vallis, 2002: The structure and composition of the annular modes in an aquaplanet general circulation model. J. Atmos. Sci., 59, 3399-3414.

Chao, W. C., and B. Chen, 2004: Single and double ITCZ in an aqua-planet model with constant sea surface temperature and solar angle. Climate Dyn., 22, 447-459.

Charney, J. G., 1947: The dynamics of long waves in a baroclinic westerly current. J. Meteor, 4, 135-163.

Charney, J. G., 1971: Tropical cyclogenesis and the formation of the intertropical convergence zone. Mathematical Problems in the Geophysical Sciences. W. H. Reid (ed.), Lectures in Applied Mathematics. 13, 355-368. Published by the American Mathematical Society.

Charney, J., 1974: Reply. J. Atmos. Sci., 31, 834-835.

Charney, J. G., and P. G. Drazin, 1961: Propagation of planetary-scale disturbances from the lower into the upper atmosphere, J. Geophys. Res., 66, 83-109.

Chen, M., and J. R. Bates, 1996: A comparison of climate simulations from a semi-Lagrangian and an Eulerian GCM. J. Climate, 9, 1126-1149.

Chen, M., R. B. Rood, and L. L. Takacs, 1997: Impact of a semi-Lagrangian and an Eulerian dynamical core on climate simulations. J. Climate, 10, 2374-2389.

Eady, E. T., 1949: Long waves and cyclone waves. Tellus, 1, 33-52.

Edmon Jr., H. J., B. J. Hoskins, and M. E. McIntyre, 1980: Eliassen-Palm cross sections for the troposphere. $J$. Atmos. Sci., 37, 2600-2616.

Egger, J., and J. Pelkowski, 2008: The first mathematical models of dynamic meteorology: the Berlin Prize contest of 1746. Meteorologische Zeitschrift, 17, 83-91.

Eliassen, A., and E. Palm, 1961: On the transfer of energy in stationary mountain waves. Geofys. Publ., 22, 1-23.

Feldstein, S., and S. Lee, 1996: Mechanisms of zonal index variability in an aquaplanet GCM. J. Atmos. Sci., 53, 3541-3556.

Frierson, D. M. W., I. M. Held, and P. Zurita-Gotor, 2006: A gray-radiation aquaplanet moist GCM. Part I: Static stability and eddy scale. J. Atmos. Sci., 63, 2548-2566.

Gates, W. L., 1992: AMIP: The Atmospheric Model Inter- comparison Project. Bull. Amer. Meteor. Soc., 73, 1962-1979.

Gates, W. L., J. S. Boyle, C. Covey, C. G. Dease, C. M. Doutriaux, R. S. Drach, M. Fiorino, P. J. Gleckler, J. J. Hnilo, S. M. Marlais, T. J. Phillips, G. L. Potter, B. D. Santer, K. R. Sperber, K. E. Taylor, and D. N. Williams, 1999: An overview of the results of the Atmospheric Model Intercomparison Project (AMIP I). Bull. Amer. Meteor. Soc., 80, 29-55.

Gill, A. E., 1980: Some simple solutions for heat-induced tropical circulation. Quart. J. Roy. Meteor. Soc., 106, 447-462.

Gutowski, W. J., L. E. Branscome, and D. A. Stewart, 1992: Life cycles of moist baroclinic eddies. J. Atmos. Sci, 49, 306-319.

Haigh, J. D., M. Blackburn, and R. Day, 2005: The response of tropospheric circulation to perturbations in lower-stratospheric temperature. J. Climate, 18, 36723685.

Hayashi, Y.-Y., and A. Sumi, 1986: The 30-40 day oscillations simulated in an "aqua planet" model. J. Meteor. Soc. Japan, 64, 451-467.

Held, I. M., 2001: The partitioning of the poleward energy transport between the tropical ocean and atmosphere. J. Atmos. Sci., 58, 943-948.

Held, I. M., 2005: The gap between simulation and understanding in climate modeling. Bull. Amer. Meteor. Soc., 86, 1609-1614.

Held, I. M., and A. Y. Hou, 1980: Nonlinear axially symmetric circulations in a nearly inviscid atmosphere. J. Atmos. Sci., 37, 515-533.

Held, I. M., and M. J. Suarez, 1994: A proposal for the intercomparison of the dynamical cores of atmospheric general circulation models. Bull. Amer. Meteor. Soc., 75, 1825-1830.

Hess, P. G., D. S. Battisti, and P. J. Rasch, 1993: Maintenance of the intertropical convergence zones and the largescale tropical circulation on a water-covered Earth. $J$. Atmos. Sci., 50, 691-713.

Holton, J. R., 1974: Comments on "Movable CISK". J. Atmos. Sci., 31, 833-834.

Holton, J. R., J. M. Wallace, and J. A. Young, 1971: On boundary layer dynamics and the ITCZ. J. Atmos. Sci., 28, 275-280.

Hoskins, B. J., 1978: Linear and non-linear baroclinic instability on the sphere. "The General Circulation: Theory, Modelling, and Observations". NCAR Summer Colloquium, Notes, NCAR/CQ-6+1978-ASP, 116-143.

Hoskins, B. J., 1983: Dynamical processes in the atmosphere and the use of models. Quart. J. Roy. Meteor. Soc., 109, 1-21.

Hoskins, B. J., and D. J. Karoly, 1981: The steady linear response of a spherical atmosphere to thermal and orographic forcing. J. Atmos. Sci., 38, 1179-1196.

Jablonowski, C., and D. L. Williamson, 2006: A baroclinic instability test case for atmospheric model dynamical 
cores. Quart. J. Roy. Meteor. Soc., 132, 2943-2975.

James, I. N., and L. J. Gray, 1986: Concerning the effect of surface drag on the circulation of a baroclinic planetary atmosphere. Quart. J. Roy. Meteor. Soc., 112, 1231-1250.

James, I. N., and P. M. James, 1992: Spatial structure of ultralow-frequency variability of the flow in a simple atmospheric circulation model. Quart. J. Roy. Meteor. Soc., 118, 1211-1233.

Jeffreys, H., 1926: On the dynamics of geostrophic winds. Quart. J. Roy. Meteor. Soc., 52, 85-104.

Kang, S. M., I. M. Held, D. M. W. Frierson, and M. Zhao, 2008: The response of the ITCZ to extratropical thermal forcing: Idealized slab-ocean experiments with a GCM. J. Climate, 21, 3521-3532.

Kodama, C., and T. Iwasaki, 2009: Influence of the SST rise on baroclinic instability wave activity under an aqua planet condition. J. Atmos. Sci., 66, 2272-2287.

Kushner, P. J., and L. M. Polvani, 2004: Stratosphere-troposphere coupling in a relatively simple AGCM: the role of eddies. J. Climate, 17, 629-639.

Lee, S., and S. Feldstein, 1996: Two types of wave breaking in an aquaplanet GCM. J. Atmos. Sci., 53, 842-857.

Lee, S., and I. M. Held, 1993: Baroclinic wave packets in models and observations. J. Atmos. Sci., 50, 14131428 .

Lee, M.-I., M. J. Suarez, I.-S. Kang, I. M. Held, and D. Kim, 2008: A moist benchmark calculation for atmospheric general circulation models. J. Climate, 21, 4934-4954.

Lin, J.-J., 2007: The double-ITCZ problem in IPCC AR4 coupled GCMs: Ocean-atmosphere feedback analysis. J. Climate, 20, 4497-4525.

Lindzen, R. S., and A. Y. Hou, 1988: Hadley circulations for zonally averaged heating centered off the equator. $J$. Atmos. Sci., 45, 2416-2427.

Lorenz, E. N., 1955: Available potential energy and the maintenance of the general circulation. Tellus, 7, 157-167.

Lorenz, E. N., 1967: The nature and theory of the general circulation of the atmosphere. WMO, No. 218, TP 115. Geneva. 161pp.

Lu, J., G. Chen, and D. M. W. Frierson, 2010: The position of the midlatitude storm track and eddy-driven westerlies in aquaplanet AGCMs. J. Atmos. Sci., 67, 3984-4000.

Matsuno, T., 1966: Quasi-geostrophic motions in the equatorial area. J. Meteor. Soc. Japan, 44, 25-43.

Mechoso, C. R., A. W. Robertson, N. Barth, M. K. Davey, P. Delecluse, P. R. Gent, S. Ineson, B. Kirtman, M. Latif, H. Le Treut, T. Nagai, J. D. Neelin, S. G. H. Philander, J. Polcher, P. S. Schopf, T. Stockdale, M. J. Suarez, L. Terray, O. Thual, and J. J. Tribbia, 1995: The seasonal cycle over the tropical Pacific in coupled ocean-atmosphere general circulation models. Mon. Wea. Rev., 123, 2825-2838.

Meehl, G. A., C. Covey, T. Delworth, M. Latif, B. McAvaney, J. F. B. Mitchell, R. J. Stouffer, and K. E. Taylor, 2007: The WCRP CMIP3 multi-model dataset: A new era in climate change research. Bull. Amer. Meteor. Soc., 88, 1383-1394.

Neale, R. B., and B. J. Hoskins, 2000a: A standard test for AGCMs and their physical parameterizations. I: The proposal. Atmos. Sci. Lett., 1, 101-107.

Neale, R. B., and B. J. Hoskins, 2000b: A standard test for AGCMs and their physical parameterizations. II: Results for the Met Office model. Atmos. Sci. Lett., 1, 108-114.

Nilsson, J., and K. A. Emanuel, 1999: Equilibrium atmospheres of a two-column radiative-convective model. Quart. J. Roy. Meteor. Soc., 125, 2239-2264.

Numaguti, A., 1993: Dynamics and energy balance of the Hadley circulation and the tropical precipitation zones: Significance of the distribution of evaporation. J. Atmos. Sci., 50, 1874-1887.

Palmer, T. N., F. J. Doblas-Reyes, A. Weisheimer, and M. J. Rodwell, 2008: Toward seamless prediction: Calibration of climate change projections using seasonal forecasts. Bull. Amer. Meteor. Soc., 89, 459-470.

Phillips, N. A., 1956: The general circulation of the atmosphere: A numerical experiment. Quart. J. Roy. Meteor. Soc., 82, 123-164.

Phillips, T. J., G. L. Potter, D. L. Williamson, R. T. Cederwall, J. S. Boyle, M. Fiorino, J. J. Hnilo, J. G. Olson, S. Xie, and J. J. Yio, 2004: Evaluating parameterizations in general circulation models: Climate simulation meets weather prediction. Bull. Amer. Meteor. Soc., 85, 1903-1915.

Polvani, L. M., and P. J. Kushner, 2002: Tropospheric response to stratospheric perturbations in a relatively simple general circulation model. Geophys. Res. Lett., 29, 18-1-18-4, doi:10.1029/2001GL014284.

Polvani, L. M., R. K. Scott, and S. J. Thomas, 2004: Numerically converged solutions of the global primitive equations for testing the dynamical core of atmospheric GCMs. Mon. Wea. Rev., 132, 2539-2552.

Raymond, D. J., and. X. Zeng, 2000: Instability and largescale circulations in a two-column model of the tropical troposphere. Quart. J. Roy. Meteor. Soc., 126, 3117-3135.

Robinson, W. A., 1991: The dynamics of the zonal index in a simple model of the atmosphere. Tellus, 43, 295-305.

Saulière, J., D. J. Brayshaw, B. J. Hoskins, and M. Blackburn, 2012: Further investigation of the impact of idealised continents and SST distributions on the northern hemisphere storm tracks. J. Atmos. Sci., 69, 840-856.

Schneider, E. K., 1977: Axially symmetric steady-state models of the basic state for instability and climate studies. Part II. Nonlinear calculations. J. Atmos. Sci., 34, 280-296.

Schneider, E. K., 2002: Understanding differences between the equatorial Pacific as simulated by two coupled GCMs. J. Climate, 15, 449-469.

Shutts, G. J., 2006: Upscale effects in simulations of tropical convection on an equatorial beta-plane. Dyn. Atmos. 
Oceans, 42, 30-58.

Simmons, A. J., and B. J. Hoskins, 1978: The life cycles of some nonlinear baroclinic waves. J. Atmos. Sci., 35, 414-432.

Sobel, A. H., and C. S. Bretherton, 2000: Modeling tropical precipitation in a single column. J. Climate, 13, 4378-4392.

Stephens, G. L., S. van den Heever, and L. Pakula, 2008: Radiative-convective feedbacks in idealized states of radiative-convective equilibrium. J. Atmos. Sci., 65, 3899-3916.

Sumi, A., 1992: Pattern formation of convective activity over the aqua-planet with globally uniform sea surface temperature (SST). J. Meteor. Soc. Japan, 70, 855-876.

Taylor, K. E., R. J. Stouffer, and G. A. Meehl, 2009: A Summary of the CMIP5 Experiment Design. Programme for Climate Model Diagnosis and Intercomparison. 33 pp. [Available at http://cmip-pcmdi.llnl.gov/cmip5/docs/ Taylor_CMIP5_design.pdf.]

Tomas, R. A., J. R. Holton, and P. J. Webster, 1999: The influence of cross-equatorial pressure gradients on the location of near-equatorial convection. Quart. J. Roy. Meteor. Soc, 125, 107-1127.

Waliser, D. E., and R. C. J. Somerville, 1994: Preferred latitudes of the intertropical convergence zone. J. Atmos. Sci., 51, 1619-1639.

Wheeler M. C., and G. N. Kiladis, 1999: Convectively-coupled equatorial waves: analysis of clouds and temperature in the wave-number-frequency domain. J. Atmos. Sci. 56, 374-399.

Williamson, D. L., M. Blackburn, B. J. Hoskins, K. Nakajima, W. Ohfuchi, Y. O. Takahashi, Y.-Y. Hayashi, H. Nakamura, M. Ishiwatari, J. McGregor, H. Borth, V.
Wirth, H. Frank, P. Bechtold, N. P. Wedi, H. Tomita, M. Satoh, M. Zhao, I. M. Held, M. J. Suarez, M.-I. Lee, M. Watanabe, M. Kimoto, Y. Liu, Z. Wang, A. Molod, K. Rajendran, A. Kitoh, and R. Stratton, 2012: The APE Atlas. NCAR Technical Note NCAR/ TN-484+STR. National Center for Atmospheric Research,Boulder,Colorado, xxii+508pp, doi:10.5065/ D6FF3QBR. [Available at http://nldr.library.ucar.edu/ repository/collections/TECH-NOTE-000-000-000865.]

Williamson, D. L., J. B. Drake, J. J. Hack, R. Jakob, and P. N. Swarztrauber, 1992: A standard test set for numerical approximations to the shallow water equations in spherical geometry. J. Comp. Phys., 102, 211-224.

Williamson, D. L., and J. G. Olson, 1998: A comparison of semi-Lagrangian and Eulerian polar climate simulations. Mon. Wea. Rev., 126, 991-1000.

Williamson, D. L., and J. G. Olson, 2003: Dependence of aqua-planet simulations on time step. Quart. J. Roy. Meteor. Soc., 129, 2049-2064.

Williamson, D. L., J. G. Olson, and B. A. Boville, 1998: A Comparison of semi-Lagrangian and Eulerian tropical climate simulations. Mon. Wea. Rev., 126, 1001-1012.

Xie, S.-P., and N. Saiki, 1999: Abrupt onset and slow seasonal evolution of summer monsoon in an idealized GCM simulation. J. Meteor. Soc. Japan, 77, 949-968.

Yang, G.-Y., B. J. Hoskins, and J. M. Slingo, 2007: Convectively coupled equatorial waves. Part I: Horizontal and vertical structures. J. Atmos. Sci., 64, 3406-3423.

Yu, J.-Y., and D. L. Hartmann, 1993: Zonal flow vacillation and eddy forcing in a simple GCM of the atmosphere. J. Atmos. Sci., 50, 3244-3259. 